\title{
Determination of the Photometric Quantities by the Silicon Photodiode Self-calibration Using Lasers
}

\author{
Yoshihiro OHNO \\ Lighting Research Laboratory \\ Matsushita Electric Industrial Co., Ltd. \\ 3-15 Yakumo-nakamachi Moriguchi, Osaka 570 Japan \\ Received 20 August 1991, in final form, 17 December 1991.
}

\begin{abstract}
The absolute spectral responsivity of inversion layer silicon photodiodes has been self-calibrated on four wavelengths using lasers, and interpolated over the entire visible region. Using the calibrated photodiodes in combination with a $V(\lambda)$ filter and a precision aperture, a photometric standard detector has been constructed. The spectral transmittance of the filter was measured by a method which can eliminate, in principle, the errors caused by the interreflections between the filter surface and the photodiode surface. The calibrated detector was compared with an illuminance standard lamp calibrated at ETL (Electrotechnical Laboratory) in Japan, and also with luminous intensity standard lamps maintained at NIST of the U.S. The luminous intensity measured by the detector agreed with the values of ETL and NIST within a $0.5 \%$ difference.
\end{abstract}

\section{Introduction}

The silicon photodiode self-calibration technique ${ }^{1)}$ has been established as a high-accuracy radiometric standard. Good results were obtained in the recent international intercomparisons of the detector absolute responsivity, in which the self-calibration technique was adopted by many laboratories $^{23}{ }^{3)}$. The wavelength region in which this technique is applied has been extended, and an uncertainty of only a few tenths of a percent in nearly the entire visible region was reported ${ }^{4 / 5)}$. These results imply the possibility of a high-accuracy determination of the photometric quantities based on this technique.

The detector-based determination of photometric quantities, such as luminous intensity or luminous flux, has long been conducted using thermal absolute radiometers since the new definition of the candela was adopted in $1979^{6}$. The uncertainty obtained in this approach, however, is on the order of $\pm 1 \%$ level $^{7)}$ in general, and a reliable method with higher accuracy is desired. As one of the approaches for this aim, the silicon photodiode self-calibration technique was applied ${ }^{8 / 9)}$ 10).

Bruening ${ }^{8)}$ determined spectral irradiance and then the luminous intensity of a lamp, using QED200 radiometer ${ }^{11)}$ and interference filters. This method requires laborious procedures for determining the spectral irradiance. Eppeldauer ${ }^{9)}$ reported an attempt to determine luminous intensity by a photometric detector whose absolute spectral responsivity was measured against a self-calibrated inversion layer type photodiode. Yoshida et al attempted to selfcalibrate a single silicon photodiode using a monochromator over the entire visible region ${ }^{12)}$, and to determine the luminous intensity of a lamp using the self-calibrated photodiode and a $V(\lambda)$ filter ${ }^{10)}$. In this approach, reproducibility of the measurements using low level monochromator radiation were to be improved. Possible error factors e. g. in the filter transmittance measurements were also to be further investigated.

As an alternative method for a detector-based determination of the photometric quantities, a method based on the silicon photodiode self-calibration using lasers on four wavelengths and the precise measurement of the spectral transmittance of the $V(\lambda)$ filter is reported in this paper. A photometric standard detector was constructed using a self-calibrated photodiode, a V $(\lambda)$ filter, and a precision aperture. Possible error factors were analyzed, and methods to minimize possible errors are proposed. The scale determined by the photometric standard detector was compared with a standard lamp calibrated at ETL of Japan and standard lamps maintained at NIST of the U.S., and the results are reported.

2. Principles of the photometric calibration based on the self-calibration technique.

The absolute spectral responsivity $R(\lambda)[\mathrm{A} / \mathrm{W}]$ of 
a silicon photodiode is expressed by ${ }^{17}$

$$
R(\lambda)=\{1-\rho(\lambda)\} \lambda \cdot \varepsilon(\lambda) / \mathrm{k}[\mathrm{A} / \mathrm{W}]
$$

where $\mathrm{k}=1239.85\left[\mathrm{~nm} \cdot \mathrm{W} \cdot \mathrm{A}^{-1}\right], \rho(\lambda)$ is the surface reflectance, $\varepsilon(\lambda)$ is the internal quantum efficiency of the photodiode. $\varepsilon(\lambda)$ is obtained by measuring the recombination losses $1-\varepsilon_{\mathrm{R}}(\lambda)$ in the rear region and 1 $-\varepsilon_{0}(\lambda)$ in the front region of a silicon photodiode, as expressed in

$$
\varepsilon(\lambda)=\varepsilon_{\mathrm{R}}(\lambda) \varepsilon_{0}(\lambda) /\left\{1-\left(1-\varepsilon_{\mathrm{R}}(\lambda)\right)\left(1-\varepsilon_{0}(\lambda)\right)\right\}
$$

When a detector is constructed, as shown in Fig.1, using the self-calibrated photodiode combined with a $V(\lambda)$ filter whose spectral transmittance is $\tau(\lambda)$ and an aperture having an opening area of $A\left[\mathrm{~m}^{2}\right]$, the responsivity for the photometric quantity $R_{\mathrm{I}}[\mathrm{A} / \mathrm{lx}]$ of this detector is given by

$$
R_{\mathrm{I}}=\frac{A \int_{0}^{\infty} P(\lambda) R(\lambda) \tau(\lambda) \mathrm{d} \lambda}{K_{\mathrm{m}} \int_{0}^{\infty} P(\lambda) V(\lambda) \mathrm{d} \lambda}[\mathrm{A} / \mathrm{lx}]
$$

where, $P(\lambda)$ is the relative spectral power distribution of the light source for which the detector is used. $V(\lambda)$ is the spectral luminous efficiency, and $K_{\mathrm{m}}$ is the maximum spectral efficacy $(683[\mathrm{~lm} / \mathrm{W}])$. By measuring the illuminance at a distance from a light source by this detector, the luminous intensity of the light source can be determined.

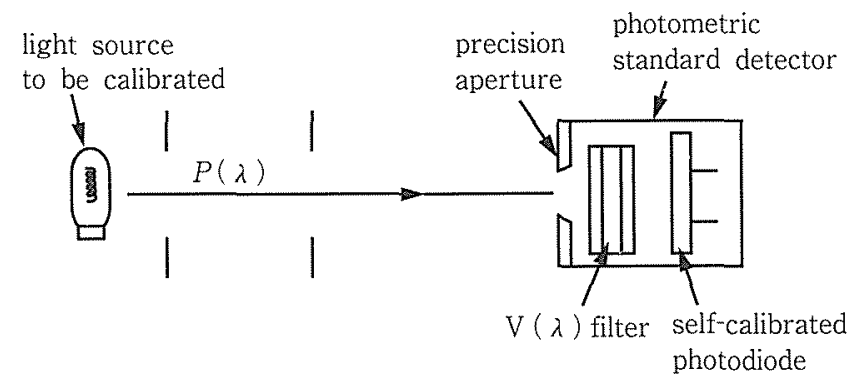

Fig.1 Luminous intensity calibration by a self-calibrated photodiode
The major error factors in determining the photometric responsivity by Eq. (3) lie in the measurements of the absolute spectral responsivity $R(\lambda)$ and the filter spectral transmittance $\tau(\lambda)$. The errors in $P(\lambda)$ will not greatly affect the value of $R_{I L}$ as long as incandescent lamps are measured, as described later. The area of the aperture opening can be measured mechanically with negligible errors. The methods for measuring $R(\lambda)$ and $\tau(\lambda)$ to minimize possible errors are described below in detail.

\section{Measurement of the absolute spectral responsivity}

3.1 Absolute responsivity self-calibration at four wavelengths

To obtain a higher accuracy in the photometric calibration with simple procedures, an attempt was made to self-calibrate photodiodes on four wavelengths of $441.6 \mathrm{~nm}, 488.0 \mathrm{~nm}$, $543.5 \mathrm{~nm}, 632.8 \mathrm{~nm}$ using small-power lasers, and to interpolate and extrapolate the absolute spectral responsivity over the entire visible region. Fig. 2 shows the configuration of the self-calibration set-up. To stabilize the beam power, acoustooptic beam stabilizers were used in combination with a monitor detector. With such systems, a measurement reproducibility of $0.05 \%$ in ten minutes was achieved. The beam power at the photodiode surface was about $0.1 \mathrm{~mW}$ except $543.5 \mathrm{~nm}(0.02 \mathrm{~mW})$ with a beam diameter of $3 \mathrm{~mm}$.

For the self-calibration sample, four inversion layer type silicon photodiodes ${ }^{13}$ UV100 (UDT) were used, with which high accuracy by self-calibration in the visible region had been previously confirmed ${ }^{5}$. This type of photodiodes (np type) can be self-calibrated only by the surface reflectance measurements with no need of the oxide bias measurements if used with enough reverse bias, and are advantageous especially when self-calibration is to be done at many wavelengths. In case of $p-n$ type photodiodes, the oxide bias measurements would be needed which cause a permanent change of the photodiode responsivity ${ }^{147}$, and one photodiode can be used for self-calibration only at one wavelength in a general method.

The results of the self-calibration measurements are shown below. Fig. 3 shows the reverse bias characteristics of a UV100 at two different power levels. Other samples showed similar characteristics. It was observed that the photo-current of all the samples saturated (the internal quantum efficiency was considered to be $100 \%$ ) at a reverse bias of $10[\mathrm{~V}]$.

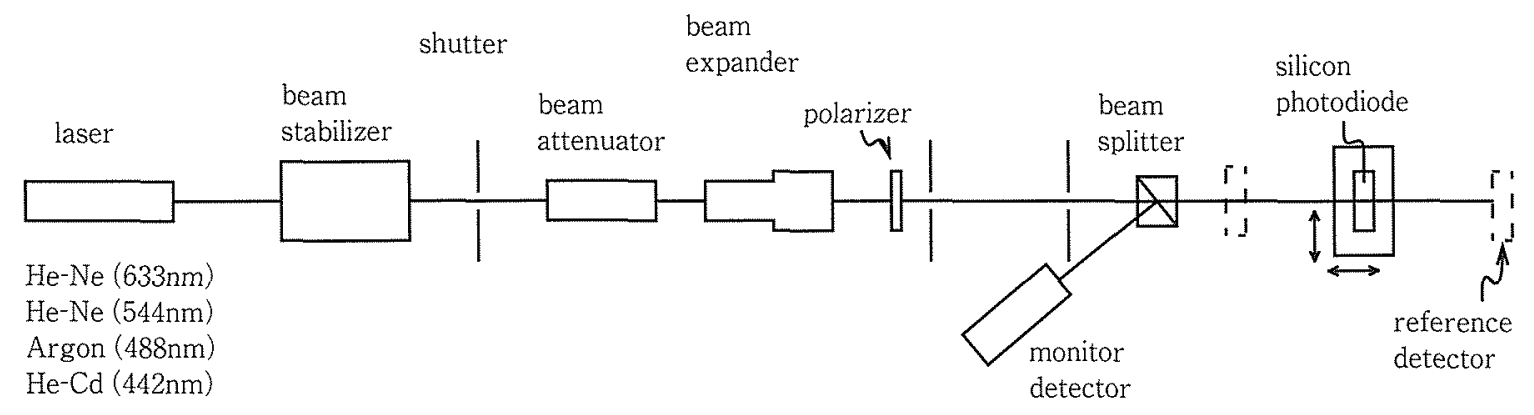

Fig.2 Optical configuration of the self-calibration set-up 


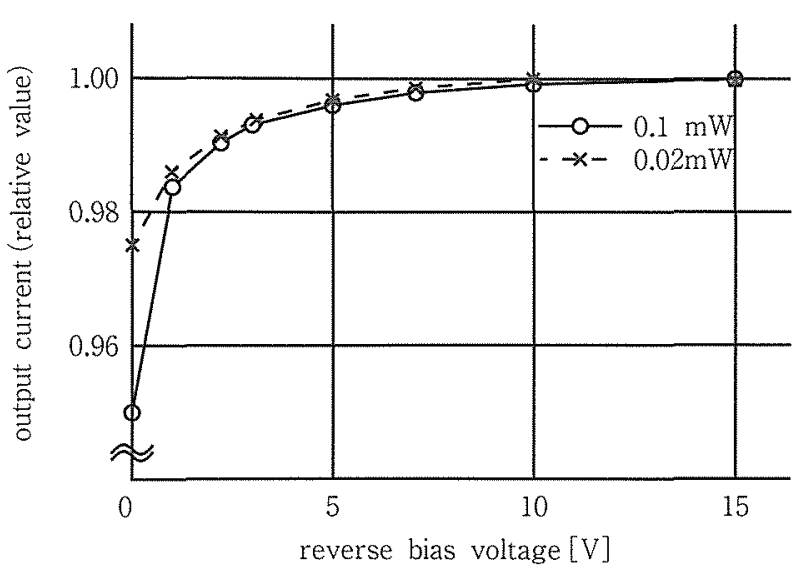

Fig.3 Reverse bias characteristics (633nm)

Each sample was used hereafter with this reverse bias voltage.

The surface reflectance of each sample was measured, and the absolute spectral responsivity $R(\lambda)$ was obtained by eq. (1). $\varepsilon(\lambda)$ was assumed to be $100 \%$ in this calculation. To verify the accuracy of this self-calibration, the absolute spectral responsivity $R^{\prime}(\lambda)$ of each photodiode was obtained by a comparison with a standard detector (two QED200 radiometers whose accuracy had been confirmed to be within $0.2 \%$ in comparison with the ESR at NIST). The results for photodiode \#1 are shown in Table 1 . The responsivities $R(\lambda)$ and $R^{\prime}(\lambda)$ of all other photodiodes at all the four wavelengths were in agreement within a $\pm 0.16 \%$ difference. These results indicated sufficient accuracy of the selfcalibration of UV100 on the four wavelengths.

Table 1 The results of the self-calibration of UV100 \#1

\begin{tabular}{c|c|c|c|c}
\hline$\lambda$ & $\rho(\lambda)$ & $R(\lambda)[\mathrm{A} / \mathrm{W}]$ & $R^{\prime}(\lambda)[\mathrm{A} / \mathrm{W}]$ & $\Delta \mathrm{R}[\%]$ \\
\hline 441.6 & 0.3579 & 0.2287 & 0.2289 & -0.07 \\
488.0 & 0.2608 & 0.2910 & 0.2911 & +0.06 \\
543.5 & 0.1720 & 0.3631 & 0.3629 & -0.07 \\
632.8 & 0.0949 & 0.4621 & 0.4619 & +0.04 \\
\hline
\end{tabular}

$\rho(\lambda)$ : surface reflectance, $R(\lambda)$ : absolute responsivity obtained by the self-calibration, $R^{\prime}(\lambda)$ : absolute responsivity measured by QED200,

$\triangle \mathrm{R}$ : difference between $\mathrm{R}(\lambda)$ and $\mathrm{R}^{\prime}(\lambda)$

3.2 Interpolation and extrapolation of the absolute spectral responsivity over the visible region

As shown in Eq. (3), in order to obtain the photometric responsivity $R_{1 L}[\mathrm{~A} / \mathrm{lm}]$, the absolute spectral responsivity $R(\lambda)$ must be obtained in the entire visible region. It is desirable that measurements be made including each end of the visible region because extrapolation is usually not accurate. Since lasers at such wavelengths were not readily available and the measured data were in the range from $442 \mathrm{~nm}$ to $633 \mathrm{~nm}$, extrapolation was made toward both ends of the visible region using a method shown below to minimize possible errors.

The surface reflectance $\rho$ of a silicon photodiode at wavelength $\lambda$ is expressed from the refractive indices $n_{1}$ of $\mathrm{SiO}_{2}, n_{2}$ of a silicon (a function of wavelength), and the thickness $d$ of $\mathrm{SiO}_{2}$ by the equation ${ }^{15}$

$$
\rho \doteqdot \frac{r_{1}^{2}+r_{2}^{2}+2 r_{1} r_{2} \cos 2 \delta}{1+r_{1}^{2}+r_{2}^{2}+2 r_{1} r_{2} \cos 2 \delta}
$$

where,

$$
\begin{aligned}
& r_{1}=\left(1-n_{1}\right) /\left(1+n_{1}\right) \\
& r_{2}=\left(n_{1}-n_{2}\right) /\left(n_{1}+n_{2}\right) \\
& \delta=2 \pi\left(n_{1} d / \lambda\right)
\end{aligned}
$$

when Eq. (5) is transformed,

$$
\begin{gathered}
d= \pm\left(\cos ^{-1} k-2 \pi i\right) \lambda /\left(4 \pi n_{1}\right) \\
(i=0,1,2 \cdots)
\end{gathered}
$$

where,

$$
k=\left\{\rho+\rho r_{1}^{2} r_{2}^{2}-\left(r_{1}^{2}+r_{2}^{2}\right)\right\}<\left\{2 r_{1} r_{2}(1-\rho)\right\}
$$

is obtained. Thus, the $\mathrm{SiO}_{2}$ thickness can be obtained from $\rho(\lambda)$. Using Eq. (5), the $\mathrm{SiO}_{2}$ thickness $d$ of each photodiode sample was calculated from the measured surface reflectance at each wavelength. Then using Eq. (4), $\rho(\lambda)$ at $380 \mathrm{~nm}$, $700 \mathrm{~nm}, 780 \mathrm{~nm}$ was calculated from $d$ obtained. Because the value of $d$ obtained varied slightly (about $1 \%$ ) depending on the wavelength, $\rho(380 \mathrm{~nm})$ was calculated using $d$ obtained from $\rho(441.6 \mathrm{~nm})$ and $\rho(\lambda)$ at $700 \mathrm{~nm}$ and $780 \mathrm{~nm}$ were calculated from $o(632.8 \mathrm{~nm})$. Fig.4 shows $\rho(\lambda)$ interpolated at every $5 \mathrm{~nm}$ over the visible region from four measured points and three calculated points. In this calculation, the refractive index of pure silicon (function of wavelength) as $n_{2}$, and the refractive index of typical $\mathrm{SiO}_{2}\left(1.47^{(6)}\right)$ as $n_{1}$ were used. Since this method is based on the optical theory of a silicon photodiode surface, it is considered to have much better accuracy than simple mathematical extrapolation. Fig.5 (the solid line) shows the absolute spectral responsivity finally obtained over the visible region from $\rho(\lambda)$ using Eq. (1).

Even though $\rho(\lambda)$ may be obtained accurately, there is a possibility that errors may increase outside the 442 $633 \mathrm{~nm}$ region where the quantum efficiency was not confirmed to be $100 \%$. To confirm this point, the relative

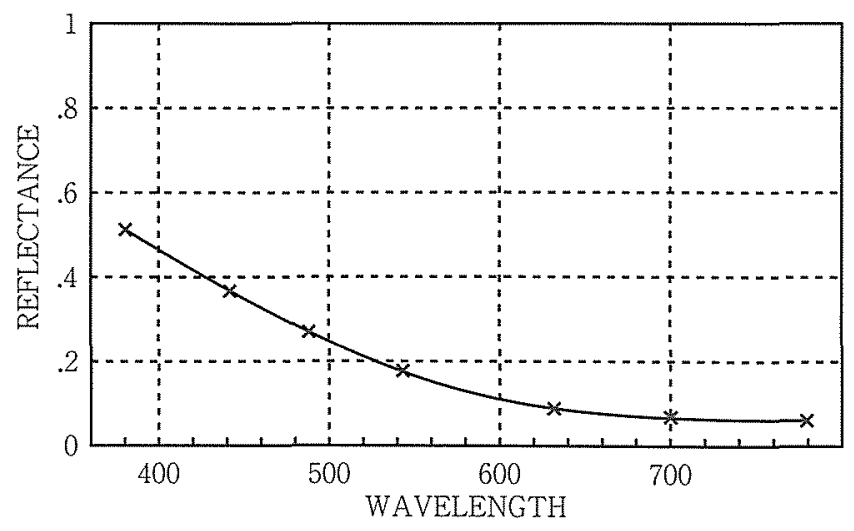

Fig.4 Extrapolated spectral reflectance of UV100 (\#1) 


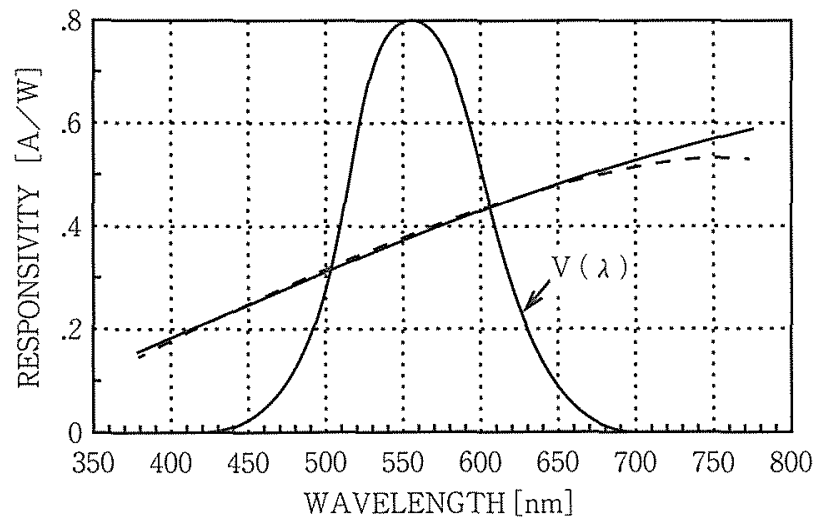

Fig.5 Absolute spectral responsivity of UV100 (\#1) obtained over the visible region (Solid line shows the data obtained by self calibration. Broken line shows the data obtained by the relative spectral responsivity measurement, normalized at $550 \mathrm{~nm}$ )

spectral responsivity of the photodiode was measured using a $\mathrm{PVF}_{2}$ detector ${ }^{16)}$ as the spectral responsivity standard. The results are shown in Fig.5 (the broken line). This curve is normalized to the solid line at $555 \mathrm{~nm}$. Considering that the accuracy of this relative spectral responsivity measurements are estimated to be about $\pm 2 \%$, the extrapolated absolute spectral responsivity agreed well with that obtained by self-calibration at wavelengths shorter than about $700 \mathrm{~nm}$. Although the difference of the two curves will increase at longer than $700 \mathrm{~nm}$, the errors in the responsivity $R_{L F}$ will be considered negligible because the $V(\lambda)$ function is nearly zero in this region.

\section{Photometric standard detector}

\subsection{Optical configuration of the detector}

A photometric standard detector was constructed using the self-calibrated photodiode. Fig. 6 shows the optical configuration of the detector. In the past approaches ${ }^{910}$, the $V(\lambda)$ filter was placed at the forefront. In the detector reported here, the precision aperture was placed at the forefront to avoid the errors caused by the change of the effective distance due to the filter's refractive index.

The diameter of the aperture opening $(6 \mathrm{~mm})$ was determined so that it would be small enough compared with the photodiode's sensitive area (10 $\mathrm{mm}$ in diameter). The precision aperture was made of stainless steel, and its opening area was measured mechanically using a precise length measuring equipment with an uncertainty of less than $1 \mu$ $\mathrm{m}$, that is an uncertainty of less than $\pm 0.03 \%$ in area.

The $V(\lambda)$ filter is composed of four glass filters (total thickness is about $10 \mathrm{~mm}$ ) so that the spectral responsivity when combined with a UV100 photodiode may be matched to the $V(\lambda)$ function (see Fig.7). The CIE $f_{1}$ ' value of the detector was about $6.5 \%$. Another feature of this detector is that the $V(\lambda)$ filter can be removed keeping the positions

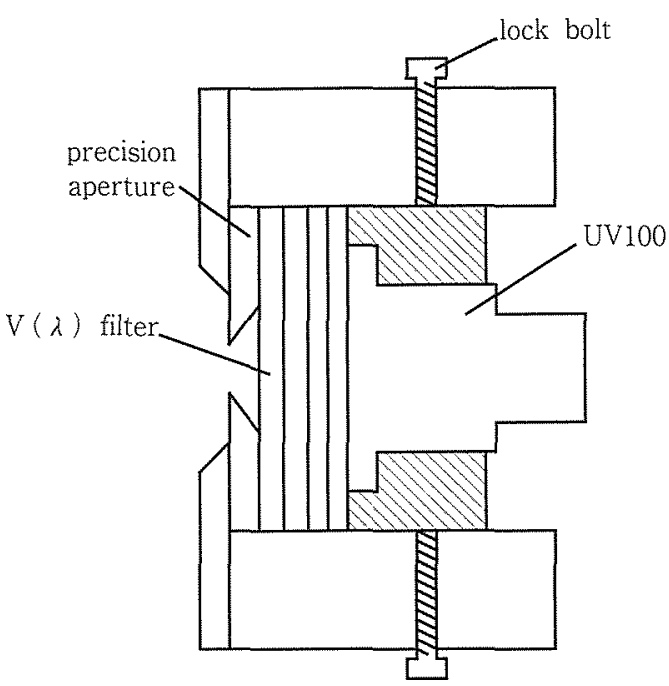

Fig.6 Optical configuration of the photometric standard detector

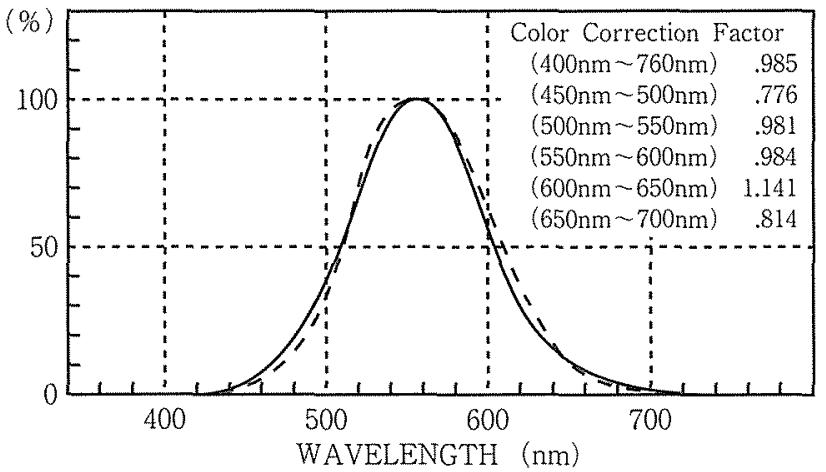

Fig.7 Relative spectral responsivity of the photometric standard detector

of the aperture and the photodiode fixed. (The reason for this is described in 5.) The photodiode can also be exchanged easily reproducing the position accurately.

4.2 Errors caused by the difference in the distribution temperature of the test lamp

The photometric responsivity of the photometric standard detector is obtained by Eq. (3), but if the spectral distribution of the test lamp is different from $P(\lambda)$ data given in Eq. (3), a measurement error is expected because the spectral responsivity of the detector cannot be perfectly matched to $\mathrm{V}(\lambda)$. Although this error is usually negligible in the ordinary photometry for incandescent lamps with small deviation of the distribution temperature, it should be investigated for the high accuracy standard detector which is the aim of this research. Table 2 shows the color correction 
Table 2 Color correction factors for incandescent lamps with varied distribution temperatures

\begin{tabular}{cc|cc}
\hline $\begin{array}{l}\text { distrib. } \\
\text { temp. }[\mathrm{K}]\end{array}$ & C.C.F & $\begin{array}{l}\text { distrib. } \\
\text { temp. }[\mathrm{K}]\end{array}$ & C.C.F \\
\hline 2600 & 1.0024 & 2850 & 1.0000 \\
2650 & 1.0019 & 2900 & 0.9996 \\
2700 & 1.0015 & 2950 & 0.9992 \\
2750 & 1.0010 & 3000 & 0.9987 \\
2800 & 1.0005 & & \\
\hline
\end{tabular}

factors of the developed photometric standard detector, which was calculated for a $2600 \mathrm{~K}$ to $3000 \mathrm{~K}$ incandescent lamp. It shows that the color correction factor is $1 \pm 0.0005$ for $\pm 50 \mathrm{~K}$ deviation from $2856 \mathrm{~K}$ of the light source. Since the distribution temperature can be measured with much higher accuracy in general, this error of the developed detector is considered to be negligible.

\section{The spectral transmittance measurement for the $V(\lambda)$ filter}

\subsection{Measurement method to avoid the interreflection errors}

Eq. (4) shows that the photometric responsivity of the photometric standard detector can be simply determined by the absolute spectral responsivity $R(\lambda)$ and the spectral transmittance $\tau(\lambda)$ of the $V(\lambda)$ filter. However, in the actual detector as shown in Fig.6, interreflections between the photodiode surface and the filter surface will occur. This would result in a serious calibration error if $\tau(\lambda)$ is measured with the $V(\lambda)$ filter taken out of the detector. Also, if $\tau(\lambda)$ is measured separately with other instru ments like a spectrophotometer, whose beam size is usually fixed, the portion and exact position of the filter to be measured will be different from the portion actually used in the photometric standard detector. In that case, some measurement error is expected due to the spatial nonuniformity of the filter transmittance.

Therefore, a method to eliminate all those errors mentioned above is devised, in which the apparent transmittance of the filter, including the effect of interreflections, can be measured. This method uses a monochromator system shown in Fig.8. The output light from the monochromator falls on the diffuser, and the diffused monochromatic light irradiates the photometric standard detector evenly over the precision aperture area. The detector was positioned $100 \mathrm{~mm}$ away from the diffuser $(50 \mathrm{~mm}$ away resulted in slightly different data), and also, the radiated area of the diffuser was limited by an aperture of $10 \mathrm{~mm}$ in diameter so that the light falling on the detector became nearly parallel and normal.

The measurement procedures are as follows: First, remove the $\mathrm{V}(\lambda)$ filter from the detector, and measure the output current of the detector $I_{1}(\lambda)$ at each wavelength. Next, insert the $V(\lambda)$ filter into the detector and measure the output current of the detector $I_{2}(\lambda)$. Then the apparent spectral transmittance $\tau^{\prime}(\lambda)$ of the $V(\lambda)$ filter is given

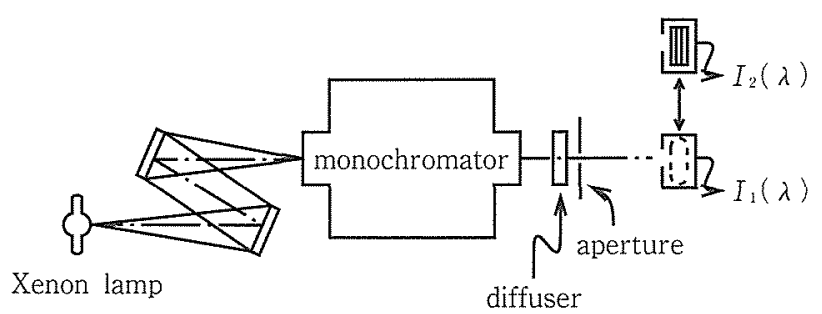

Fig.8 Measurement system for apparent spectral transmittance of the $V(\lambda)$ filter

by :

$$
\tau^{\prime}(\lambda)=I_{2}(\lambda) / I_{1}(\lambda)
$$

Throughout this measurement, the detector was remained fixed to the measurement system. The detector is made so that the $V(\lambda)$ filter could be removed from the back while the detector is fixed to the system. Once the detector is detached from the measurement system, it is usually difficult to reproduce the same position. Only a $0.5 \mathrm{~mm}$ error will result in $1 \%$ error of measured transmittance which is unacceptable in this calibration.

The UV100 photodiode used in the detector needs a reverse bias, which causes a significant dark current, whose drift may result in a serious error. Therefore, to reduce this error, a short-arc Xenon lamp (Hamamatsu, 75W) was used for the monochromator source for higher monochromator output. Also, to increase measurement accuracy, $I_{1}$ $(\lambda)$ and $I_{2}(\lambda)$ were measured five times alternately, and the average of five sets of $\tau^{\prime}(\lambda)$ data was adopted.

The measured $\tau^{\prime}(\lambda)$ is shown in Fig.9 (the solid line). The $\tau(\lambda)$ data measured with a spectrophotometer (Hitachi U3400) is also shown in the broken line. A significant

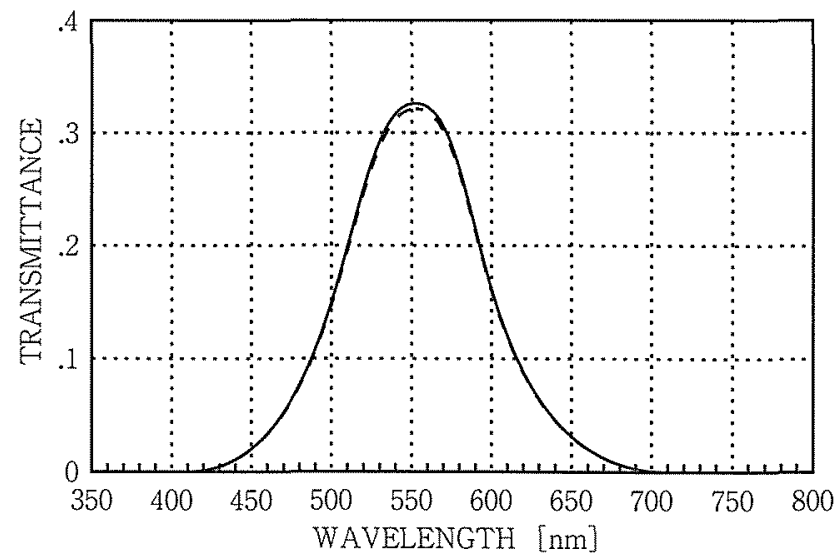

Fig.9 Spectral transmittance of the $V(\lambda)$ Filter (Solid line shows the data measured with the method shown in Fig.8. Broken line shows the data measured with a spectrophotometer) 
difference is recognized between the two curves. This difference is considered to be caused by the interreflection effect and the nonuniformity error as well as the errors of the spectrophotometer. It is evident, anyway, that measuring $\tau(\lambda)$ separately by itself will not give enough accuracy for the detector photometric responsivity. In this measure-ment, one of the photodiodes $(\# 1)$ was used for the four $V(\lambda)$ filters because the surface reflectances of the four photodiodes used were nearly equal, and the difference in the interreflection effect among the four photodiodes was considered negligible.

\subsection{Analysis of errors in the transmittance in infrared}

Incandescent lamps have larger power in the infrared region than in the visible, and a silicon photodiode has a peak responsivity around the wavelength of $1 \mu \mathrm{m}$. Therefore, only a small leak of transmittance of the $V(\lambda)$ filter in the infrared region will result in a serious error in the photometric responsivity of the photometric standard detector. If the relative spectral responsivity of the detector remained $0.1 \%$ in the $780-1100 \mathrm{~nm}$ region, and if it is ignored, an error as much as $0.8 \%$ in the photometric responsivity would be caused, which would be an unacceptable error for a high-accuracy standard detector aimed at in this research. In the interpolation of the absolute spectral responsivity mentioned in 3.2 and in the calculation of the detector's responsivity mentioned in 6. , only the data in the visible region is processed. Therefore, the relative spectral responsivity of the calibrated detector in the infrared region was measured precisely. The measurement was made using the monochromator system shown in Fig.8. The results of measurements repeated three times are shown in Fig.10. The vertical axis shows the responsivity relative to its peak in percent. From this data, the error in the detector photometric responsivity caused by the spectral responsivity in the region $780-1100 \mathrm{~nm}$ was calculated, and it was confirmed that the error was less than $0.02 \%$

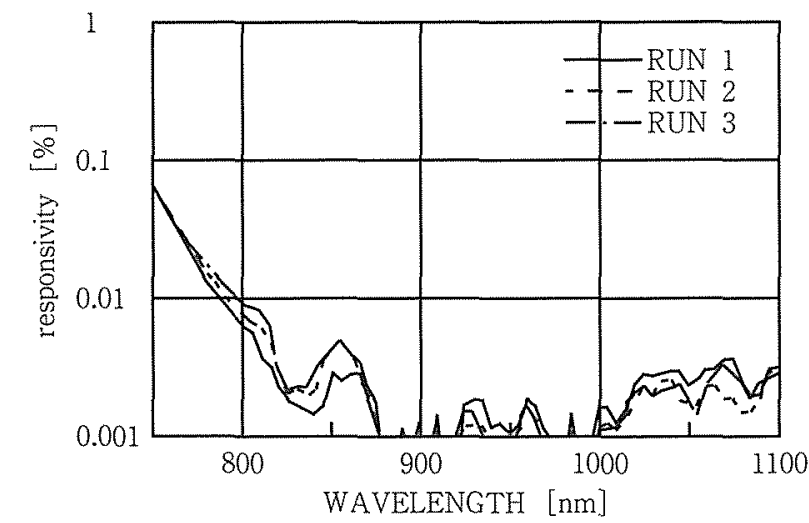

Fig.10 Relative spectral responsivity of the photometric standard detector in the infrared

\section{The uncertainty of the photometric standard detector}

As mentioned above, the absolute spectral responsivity $R(\lambda)$ of four silicon photodiodes and the apparent spectral transmittance $\tau^{\prime}(\lambda)$ of the $V(\lambda)$ filter were measured, and the photometric responsivity $R_{\mathrm{IL}}[\mathrm{A} / \mathrm{lx}]$ was calculated by Eq. (3). The results are shown in Table 3. For $P(\lambda)$, the spectral distribution of $2856 \mathrm{~K}$ black body was used. The row of Si-PD \#1 to \#4 shows each case when each photodiode \#1 to \#4 was incorporated in the detector. The value of $\sigma$ shows the standard deviation of the detector responsivities calculated from the five $\tau^{\prime}(\lambda)$ measurements described in 5 .

Table 3 The calculated responsivities of the photometric standard detector

\begin{tabular}{c|c|c|c}
\hline $\begin{array}{l}\text { self-cal. } \\
\text { Si-PD }\end{array}$ & $\begin{array}{c}\text { responsivity } \\
\times 10^{-4}[\mathrm{~A} / \mathrm{Im}]\end{array}$ & $\begin{array}{c}\text { responsivity } \\
\times 10^{-9}[\mathrm{~A} / \mathrm{Ix}]\end{array}$ & $\begin{array}{l}\sigma \\
{[\%]}\end{array}$ \\
\hline$\# 1$ & 1.7209 & 4.8660 & \\
$\# 2$ & 1.7244 & 4.8759 & 0.37 \\
$\# 3$ & 1.7104 & 4.8363 & \\
$\# 4$ & 1.7219 & 4.8688 & \\
\hline
\end{tabular}

The uncertainty of the detector is analyzed as follows. The photometric responsivity $R_{\mathrm{I} \mathrm{L}}[\mathrm{A} / \mathrm{lx}]$ of the photometric standard detector is shown in Eq. (3). This equation shows the source of errors to be in $R(\lambda)$ (the silicon photodiode absolute spectral responsivity), $\tau(\lambda)$ (spectral transmittance of the $V(\lambda)$ filter), $P(\lambda)$ (spectral distribution of the light source), and $A$ (area of the aperture). As for $R(\lambda)$, interpolation between the four wavelengths is considered to have sufficient accuracy. The extrapolated portion might not be as accurate as interpolation, but the errors in this portion is considered negligible in the photometric responsivity as shown in 3.2. Therefore, the uncertainty of $R(\lambda)$ is mainly determined by the uncertainty of the absolute responsivity self-calibration on the four wavelengths, which was within $\pm 0.3 \%{ }^{5}$. As for $\tau(\lambda)$, assuming there is no systematic errors in the method shown in 5.1 , the uncertainty of the measurement should be \pm $0.37 \%(1 \sigma)$ which was the measurement reproducibility. The measurement uncertainty of the aperture area is considered negligible as shown in 4.1. As for $P(\lambda)$, a possible error factor will be the errors associated with the color correction factor of the detector, which was analysed in 4.2 to become no serious errors for incandescent lamps to be calibrated. In addition to these factors, the reproducibility of the measurements by the detector should also be considered, which was $\pm 0.1 \%(1 \sigma)$. Assuming the above-mentioned measurement uncertainty of $R(\lambda), \tau(\lambda)$, and the reproducibility of the detector to be random errors, the total uncertainty of the photometric standard detector calibrated this time is considered to be about $\pm 0.5 \%$.

\section{Intercomparison with official standard lamps}

7.1 Comparison with an illuminance standard lamp calibrated at ETL

The luminous intensity of an illuminance standard lamp calibrated at ETL (Electrotechnical Laboratories) of Japan 
was measured by the photometric standard detector, and the measured values were compared. The illuminance standard lamp (Kondo Sylvania, monoplane type, 500W, 713cd) was calibrated at ETL in March 1989. This comparison measurement was made in the same month. Each photodiode \#1 to \#4 was installed in the detector in turn, and the luminous intensity of the standard lamp was measured at a distance of $2 \mathrm{~m}$.

Fig.11 shows the luminous intensities measured by the photometric standard detector, relative to the value determined at ETL. Measurements were repeated five times (runs). Each photodiode \#1 to \#4 was changed and measured during one run. The standard lamp was once turned off after each run. The black circles show each measured data. The white circles show the mean value of the five runs for each photodiode installed in the detector. The five runs ended up in including different measurement date, re-alignment of the standard lamp, and different turnedoff time between runs.

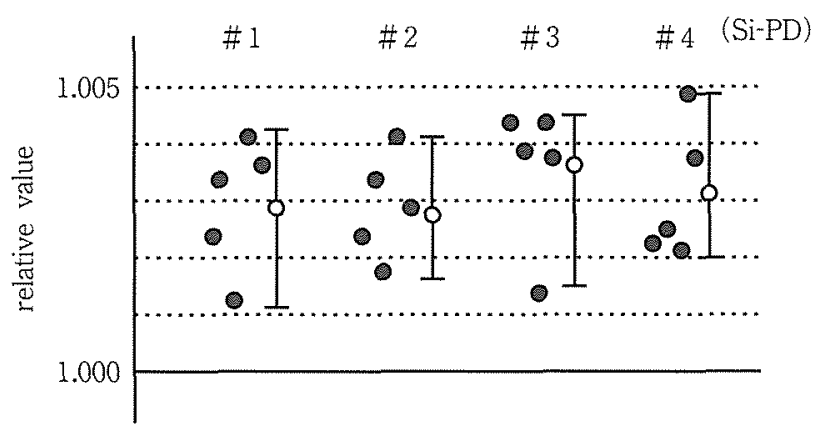

Fig.11 Luminous intensity measured by the photometric standard detector compared with the ETL value

In Fig.11, first, the mean values of the five runs for each photodiode (white circles) are in agreement within a 0.1 $\%$ difference, which indicates good reproducibility of the self-calibration measurements. On the other hand, about $0.3 \%$ deviations are found in the data of five runs for each photodiode, which can imply not very good reproducibility of the detector. However, the black circles (plotted from the left in the time order of runs) form similar patterns for each photodiode. This indicates that the deviations were caused mainly by the reproducibility of the standard lamp used, and the reproducibility of the detector may be better. The average of the values measured by the detector was in agreement with the value by ETL having about a $0.3 \%$ difference.

\subsection{Comparison with standard lamps maintained at NIST}

The photometric standard detector was brought to U.S. National Institute of Standards and Technology in October 1989, and intercomparison measurements were made with the 14 luminous intensity standard lamps maintained at NIST. These were nine NBS 5600 series lamps (gas filled, 500W, diffuse bulb), and five NBS 10400 series lamps (gas filled,
$200 \mathrm{~W}$, clear bulb). They were all calibrated from the spectral irradiance standard lamp which was based on a gold-point black body. In the detector brought, the silicon photodiode \# 1 was installed and a reverse bias of 10 [V] was applied. The amplifier used was made at NIST, whose gain setting was calibrated with $0.03 \%$ accuracy, and used with a high accuracy DVM (HP3456A). The luminous intensity of the 14 standard lamps were measured by the detector at a distance of $2 \mathrm{~m}$. Each value was taken as a mean value of ten readings of the DVM. The standard deviation of the ten readings for each lamp was checked to be less than $0.1 \%$. After all the measurements were finished, the photometric responsivity of the detector was given, and the measured luminous intensity values were calculated and compared with the values determined at NIST.

The values measured by the detector were in agreement with the 14 standard lamps in the range $-0.54 \%$ to $+0.31 \%$. The deviations of the degree of agreement are considered to be due to the uncertainty of the 14 standard lamps, and not to the reproducibility of the detector. the average of the difference between the detector's values and NIST values was about $0.1 \%$, with a standard deviation of $0.26 \%$. Before and after the detector was brought to NIST, the responsivity was checked by measuring a same luminous intensity standard lamp, and the change was found to be within $0.2 \%$.

Together with the results mentioned in 7.1, it has been confirmed that the photometric standard detector calibrated with the methods described in this paper agreed with standard lamps officially calibrated at ETL and NIST within about a $0.5 \%$ difference.

\section{Conclusion}

The absolute responsivity of a silicon photodiode was selfcalibrated using lasers on four wavelengths, and the data was interpolated and extrapolated over the entire visible region using the surface reflection theory. A photometric standard detector was constructed using the calibrated photodiode and a $V(\lambda)$ filter. The spectral transmittance of a $V(\lambda)$ filter was measured with a method that can eliminate the errors caused by the interreflections between the photodiode surface and the filter. Other possible error factors were analyzed and it was confirmed that no significant errors would be caused. The photometric responsivity of the photometric standard detector was then calculated, and the luminous intensities of standard lamps calibrated at ETL and those maintained at NIST were measured by the detector. The values measured by the detector were in agreement with the values calibrated at the national laboratories within a $0.5 \%$ difference. These results indicate that a sufficient accuracy was obtained in the photometric standard detector calibrated this time.

To reduce the overall uncertainty of the detector effectively, the reproducibility of the measurement of the spectral transmittance of the $V(\lambda)$ filter is to be improved. The temporal stability and the temperature dependence of the detector responsivity is to be investigated in order to use the photometric standard detector as a transfer standard as well as a primary standard. Also, to improve and verify 
the accuracy of the detector, it is hoped that there will be more chances for intercomparisons with many other laboratories who attempts to realize high-accuracy photometric standards.

The author would like to express his thanks to I.Saito of Electrotechnical Laboratory, Y.Mishima of Japan Luminaires Association, Y.Nakagawa of Saitama Univ. for their valuable opinions and discussions on this research. Great thanks are also due to K.D.Mielenz, D.A.McSparron and G.Eppeldauer at U.S. National Institute of Standards \& Technology for their accepting the intercomparison measurements and giving various help at the measurements.

\section{References}

(1) Zalewski, E. F. and Geist, J. : Silicon photodiode absolute spectral response self-calibration, Appl. Opt. 19-8, 1214 (1980)

(2) Zalewski, E. F. : Comparison of the National Standards of Absolute Spectral Responsivity, BIPM-CCPR-Rapport de la lle session, 165 (1986)

(3) Thomas, D. B. and Zalewski, E. F. : Results of CIE detector response intercomparisons, J. Res. Natl. Inst. Stand. Technol. 95-5, 535 (1990)

(4) Gardner, J.L. and Brown, W. J. : Silicon radiometry compared with the Australian radiometric scale, Appl. Opt. 26-12, 2431 (1987)

(5) Ohno, Y. and Nishiyama, H. : Self-calibration of the silicon photodiode absolute responsivity in the visible region and its accuracy, J. Illum. Engng. Inst. Jpn. 75-6, 314 (1991) (in Japanese)

(6) CIE Publ. No.18. 2, (TC1.2), The Basis of Physical Photometry (1983)

(7) Bonhoure, J. : Sixth comparison of national standards of luminous intensity and of luminous flux (1985), Metrologia 24, 157 (1987)

(8) Bruening, R. J. : Spectral irradiance scales based on filtered absolute silicon photodetectors, Appl. Opt. 26-6, 1051 (1987)

(9) Eppeldauer, G. : Longterm changes of silicon photodiodes and their use for photometric standardization, Appl. Opt. 29-5, 2289 (1990)

(10) Yoshida, M., Nishiyama, H. and Yoshie, K. : Measurement of luminous intensity by the self-calibration technique, J. Illum. Engng. Inst. Jpn. 71-10, 618 (1987) (in Japanese)

(11) Zalewski, E. F. and Duda, C. R. : Silicon photodiode device with $100 \%$ external quantum efficiency, Apply. Opt. 2218, 2867 (1983)

(12) Tsuruoka, H. and Yoshie, K. : Estimation of absolute spectral sensitivity of a silicon photodiode, J. Illum. Engng. Inst. Jpn. 69-10, 531 (1985) (in Japanese)

(13) Booker, R. L. and Geist, J. : Induced junction (inversion layer) photodiode self-calibration, Appl. Opt. 23-12, 1941 (1984)

(14) Key, P. J., Fox, N. P. and Rastello, M. L. : Oxide-bias measurements in the silicon photodiode self-calibration technique, Metrologia, 21, 81 (1985)

(15) Huen, T. : Reflectance of thinly oxidized silicon at normal incidence, Appl. Opt. 18-12, 1927 (1979)

(16) Nakagawa, Y. and Otani, Y. : Precise measurements of the spectral sensitivity of radiation detectors in the UV and VIS. Region (1), J. Illum. Engng. Inst. Jpn. 66-10, 436 (1982) (in Japanese) 